\title{
Intelligent System to Analyze Data about Powered Wheelchair Drivers
}

\author{
Malik Haddad ${ }^{1}$, David Sanders ${ }^{1}$, Martin Langner ${ }^{2}$, Mohamad Thabet ${ }^{1}$, Peter \\ Omoarebun $^{1}$, Alexander Gegov ${ }^{1}$ \\ ${ }^{1}$ University of Portsmouth, Portsmouth PO1 3DJ, UK \\ ${ }^{2}$ Chailey Heritage Foundation, North Chailey, BN8 4E, UK \\ david.sanderseport.ac.uk
}

\begin{abstract}
The research presented in this paper creates an intelligent system that collects powered wheelchair users' driving session data. The intelligent system is based on a Python programming platform. A program is created that will collect data for future analysis. The collected data considers driving session details, the ability of a driver to operate a wheelchair, and the type of input devices used to operate a powered wheelchair. Data is collected on a Raspberry Pi microcomputer and is sent after each session via email. Data is placed in the body of the emails, in an attached file and saved on microcomputer memory. Modifications to the system is made to meet confidentiality and privacy concerns of potential users. Data will be used for future analysis and will be considered as a training data set to teach an intelligent system to predict future path patterns for different wheelchair users. In addition, data will be used to analyze the ability of a user to drive a wheelchair, and monitor users' development from one session to another, compare the progress of various users with similar disabilities and identify the most appropriate input device for each user and path.
\end{abstract}

Keywords: Analysis, Disabled, Intelligent System, Data Collection, Python Programming Language, Powered Wheelchair, Raspberry Pi.

\section{Introduction}

Research presented in this paper is part of a larger research project at the University of Portsmouth [1]. The main aims are to use AI to enhance mobility and improve the quality of life of disabled powered wheelchair users through increasing their selfconfidence and reliance.

Demographic figures in the United Kingdom (UK) estimated the UK population in 2020 above 67 million individuals. A Family Resources Survey in Great Britain, conducted by the Department for Work and Pension 2016/17 claimed that around 13.9 million individuals of the UK population suffered from some kind of disability, and $51 \%$ of them had a mobility problem [2].

Assistive devices often compensate any loss of ability and on-going deterioration in the individual mobility over time while decreasing the dependency on carers [3]. People using powered wheelchairs can be missing the required cognitive/motor/sensory 
ability, because of disease, disability with their hands, arms, shoulders or even more extensive disabilities [4].

People with visual impairment or people who are not able to take any avoiding action by themselves are potential new users for the new systems described in this paper. The new systems can assist new powered wheelchair users with the navigation of their wheelchairs.

A smart wheelchair is a powered wheelchair with computers and sensors attached to it. A disabled user specifies a desired speed and bearing by placing or moving a transducer into a position. For example, using switches or a joystick. A powered wheelchair will then have a tendency to progress along the route specified (and to move at the anticipated speed). A user can then make adjustments to avoid objects. Fig. 1 shows an example of a wheelchair used in this research.

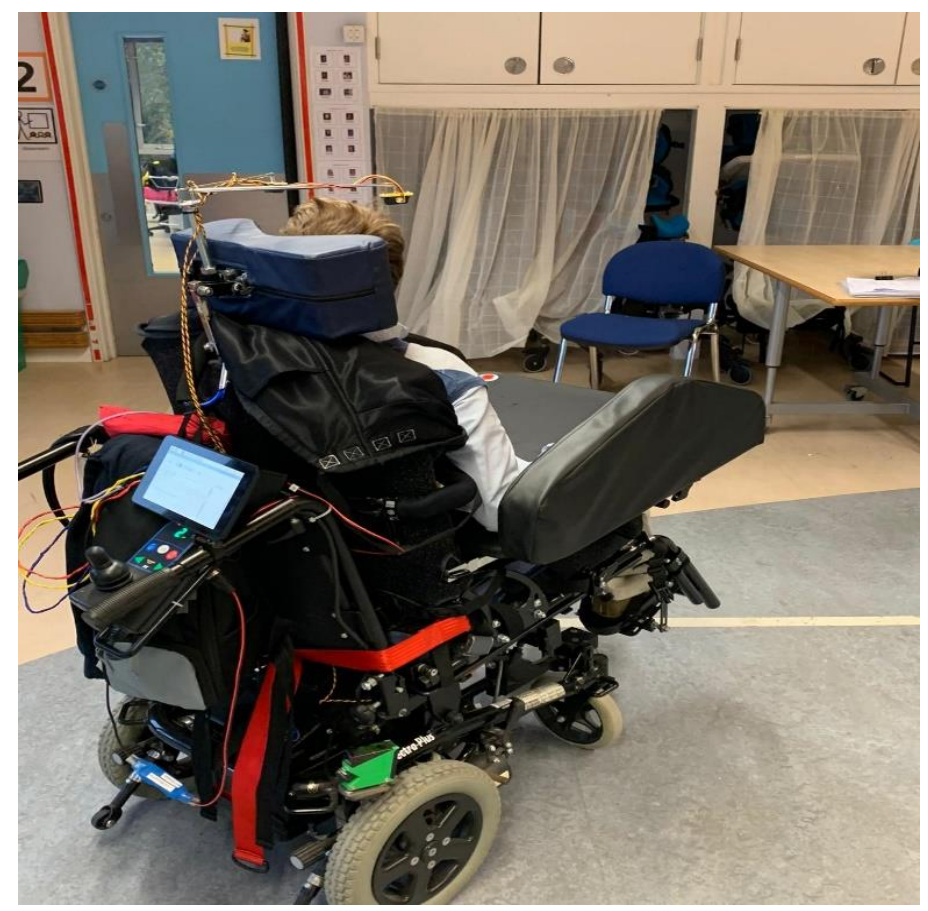

Fig. 1. Example of a wheelchair considered during the research.

The large wheel shown in Fig. 1 provided motion and direction. Each large wheel was operated independently using a separate motor. Powered wheelchair users often attend driving sessions to learn how to use input devices that control their wheelchair. Wheelchair users navigate by varying electrical current to the individual motors. Joysticks are normally used to control the velocity (direction and speed) of a powered wheelchair. If users were unable to use their fingers or hands or lacked the necessary dexterity, head or chin switches, foot control or puff/sip tubes could be considered as other input options. 
Considerable research was conducted to study the steering and navigation of powered wheelchairs [5-17]. The systems used were often local, and slight work has been made to improve mobility using more global approaches. Research has considered collision avoidance [18-23] using sensors that delivered a more local sense of the surrounding of the wheelchair [24].

Research has often suggested an initial wheelchair path that can be locally adjusted if obstructions were present, but they have rarely been successfully applied to help disabled wheelchair users. The new intelligent systems to predict potential desired routes that are under consideration and described here could rapidly predict routes.

In this paper, a technique that will gather data from users' driving sessions and the nature of data gathered is described. A Raspberry Pi microcomputer was fitted between user input devices and powered wheelchair motors to intercept and collect the required data.

A Python program was created and installed on the Raspberry Pi. The program gathered the required data. The collected data was stored on the Raspberry Pi memory, and at the end of each driving session, the collected data was emailed to a specified email address. Data was sent in the body of the emails and in a Comma Separated Value (CSV) file. A graphical representation of the data in the shape of a pie-chart was created and attached to the emails. Data will be considered for future analysis and will be used as a training data set to train an intelligent system that will predict future route patterns for different wheelchair users. In addition, data will be studied to measure the ability of a user to operate their wheelchair, and users' progress from one session to another, compare the progress of different users with the same type of disability and identify the most suitable input device for each user and route.

In the work described in this paper, Raspberry Pi microcomputers were considered because of their small physical size, low cost, upgradeability, replicability and simplicity [12]. The program installed on the Raspberry Pi is described in Section 2. Section 3 provides modification to the program to meet privacy and confidentiality concerns. Section 4 is a discussion about how the collected data will be used for analysis. Section 5 presents conclusions drawn from the current system and Section 6 presents some future work.

\section{The Python Program}

The aim of the project was to create a new system that gathered users' driving session data using a simple, robust and low cost architecture. Raspberry Pi Microcomputers were considered because they are often considered simple and robust computers with reasonable price. They were first launched in 2012 with an option of two models: A and B. In 2014, Model B+ was launched that included hardware improvements compared to the previous models [25]. The research conducted in this paper considered a Raspberry Pi model 3B+.

The Python programming language was used to create a program that will gather required driving session data for future analysis. The program was installed onto a 
Raspberry Pi. The Raspberry Pi digitized users desired input. The desired direction supplied by the user was saved on the Raspberry Pi memory.

A first step towards creating the new program was to create a new User Interface (UI) to simplify the interaction with helpers and carers, the UI requested a helper/carer to give the following details at the beginning of a driving session as shown in Fig. 2:

- User name.

- Name of the input device used.

- Any medication that could impair the user driving ability.

- When the last dose (of medication) was administered hh:mm.

- When next dose (of medication) will be administered hh:mm.

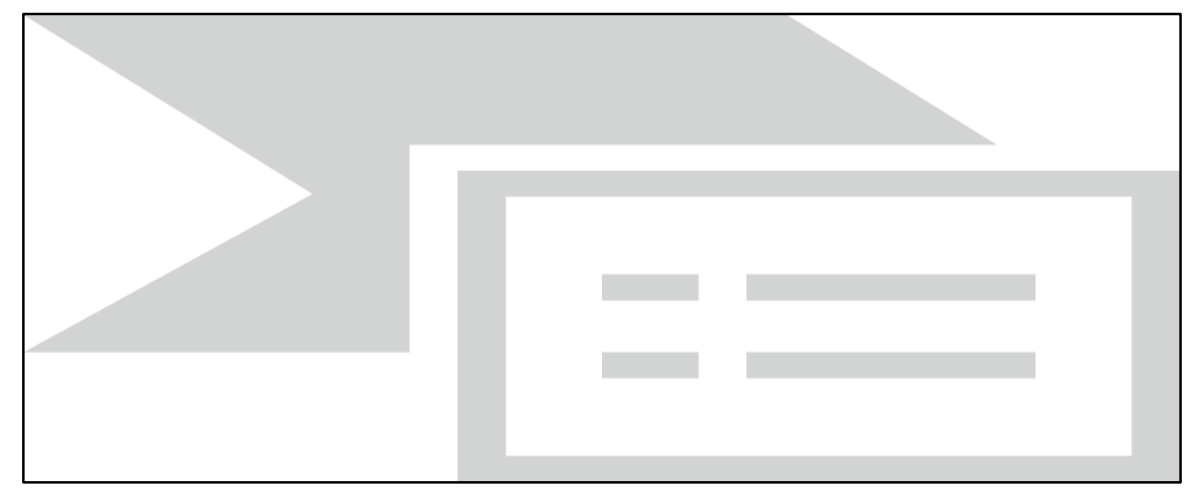

Fig. 2. User Interface for the new program.

An emergency exit function was considered if anything went out of control. If an emergency exit was requested, the Raspberry $\mathrm{Pi}$ would switch off power from wheelchair motors, save and email all the details of the driving session and state that an emergency exit was requested with the date/time of the request.

An End of Session exit function was created that switched off power from the wheelchair motors and exited the program. In case an End of Session exit was requested the program would exit, save and email all the details of the driving session and state that no emergency was required.

Data collected from emails contained the following:

- Name of the user.

- Session start time.

- Type of input device used.

- Name of any medication that could impair user ability.

- Time when the user was last administered that medication.

- Time of the next dose of that medication.

- Duration of moving backward in seconds.

- Duration of moving forward in seconds.

- Duration of moving to the left in seconds.

- Duration of moving to the right in seconds. 
- Duration when no switch was pressed.

- Order of switch presses.

- Number of time each switch was pressed.

- Whether an emergency exit was required.

- Session end time.

Fig. 3 shows a screenshot of the CSV file attached to the emails including some sample data. Fig. 4 shows a graphical display (pie-chart) for data obtained from the switches, in this example three switches were considered (Red, Yellow and Blue) which was a simple visual summary of the session.

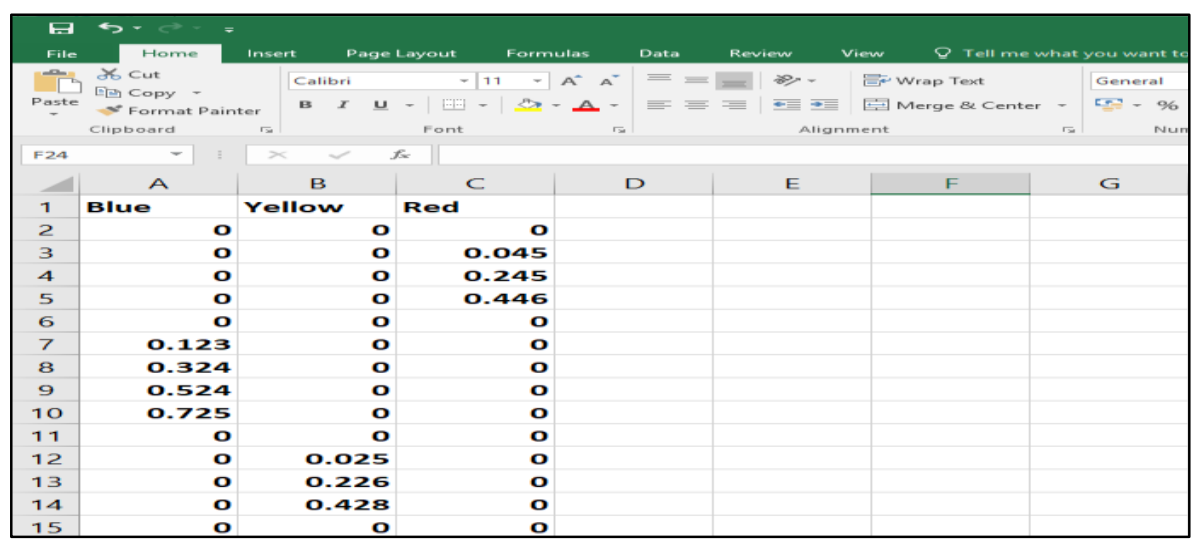

Fig. 3. Example of CSV file showing driving session data.

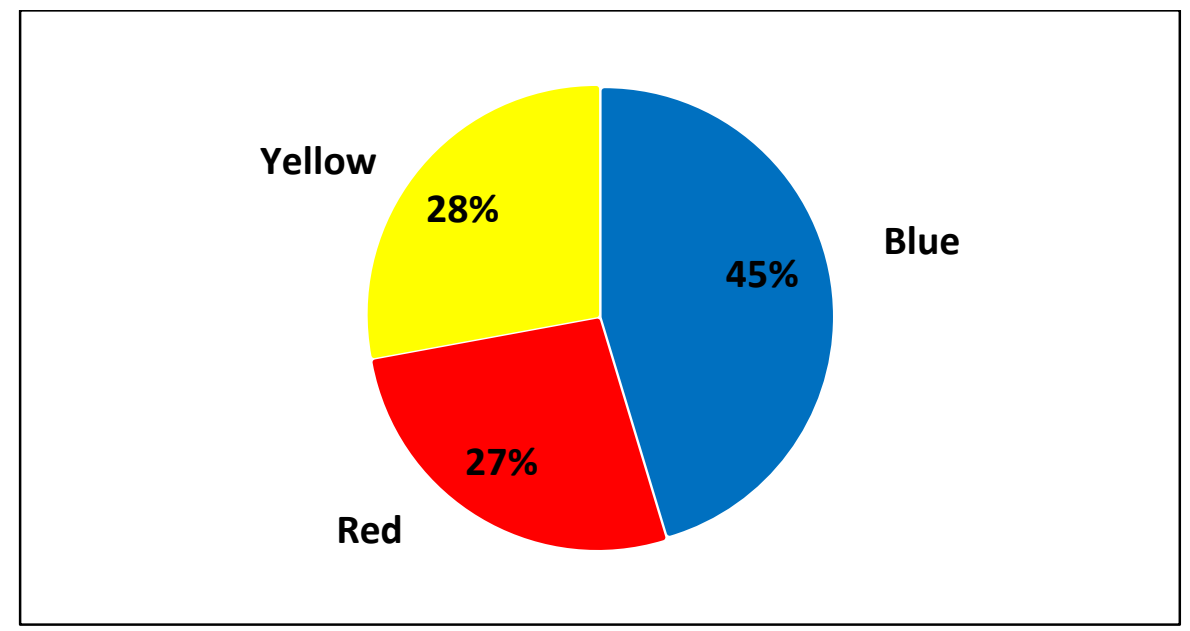

Fig. 4. Example of graphical representation (pie-chart) showing data obtained from switches. 


\section{Modification to the New System}

To meet privacy policy and confidentiality concerns of potential users, the new system was subjected to several modifications. The email function presented in the previous section was disabled and all data were stored in the microprocessor memory. Data were retrieved from the microprocessor memory on a monthly basis.

A further modification to the new system was conducted to interface two IR head switches as shown in Fig. 5. The new modified system was mounted on to a powered wheelchair used by a student at Chailey Heritage School as shown in Fig. 6. Guardian consent was acquired and the collected data will be considered for analysis and will be used as a training set for an intelligent system to predict future route patterns. In addition, data will be used to analyze the ability of the user to operate their wheelchair, and monitor user's progress from one session to another and compare the progress of different users with the same type of disability.

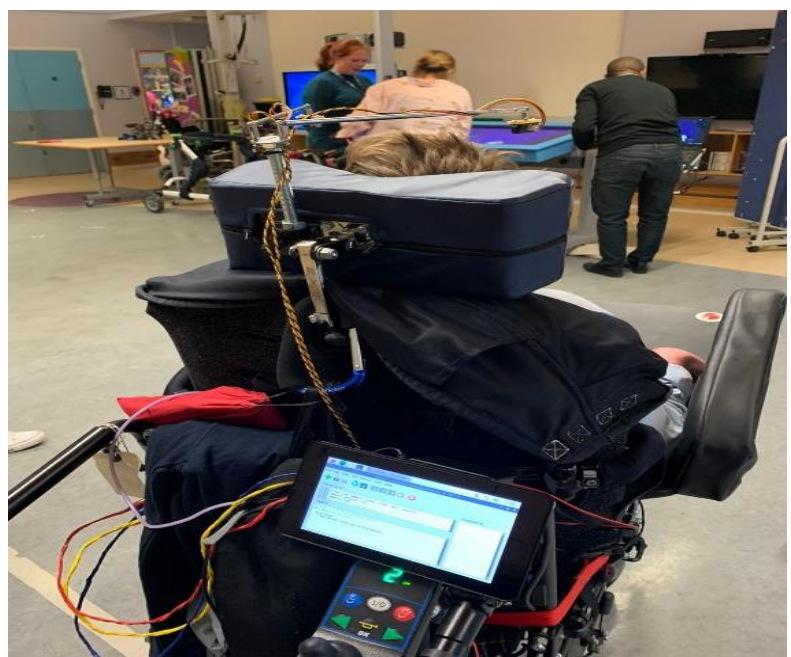

Fig. 5. Modified New system interfacing to IR head switches. 


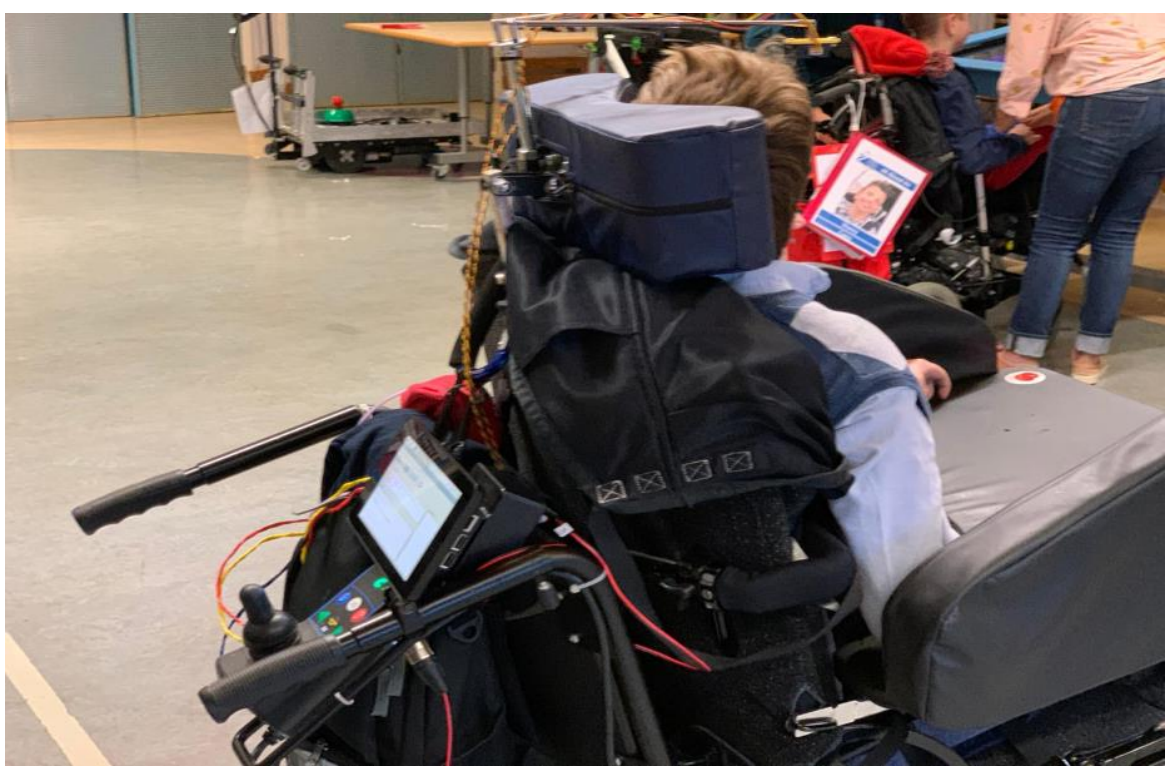

Fig. 6. Powered wheelchair using the modified new system.

\section{Discussion}

Research presented in this paper is part of a larger research project being conducted by the authors at the University of Portsmouth [1] that aims to improve mobility and enhance the quality of life of disabled powered wheelchair users by increasing their self-confidence and reliance.

This research used a Raspberry Pi to store users' driving session data and email data to a predefined email address for future reference and analysis. The new system was clinically trialled and results showed that it successfully stored session data and emailed data to a specified email address.

Data received will be used as a training set to train an intelligent system to predict routing patterns for different wheelchair users, assess users' progress from one session to another and compare the progress of the different users with similar types of disability. Data will be used to identify how different types of disability could affect user progress, what and how different types of medication could affect driving ability, identifying a "best time in the day" for each user to conduct driving session, and how driving ability was affected by the duration of driving. In addition, data will be used to estimate the time spent travelling by a user in each session, monitor user ability factors and the need for assistance and analyze which type of input device best suited each user and different route patterns. 


\section{Conclusions}

This paper presented a new system that gathered users' driving session data and could be used as an interface between any user input device and powered wheelchair motors. The work will apply simple and computationally inexpensive AI software.

A python program was created and installed on a Raspberry Pi to gather users' driving session data and save and email collected data to a predefined email address. A CSV file was created and attached to the emails containing driving session data.

A graphical representation of the session data (pie-chart) was created and attached to the emails to improve understanding of any patterns.

Modifications to the new system were applied to meet confidentiality and privacy concerns of collaborators and users.

The authors are building a friendlier user interface for the new system that could simplify the operation of a powered wheelchair and gather subjective feedback about driving sessions from helpers and carers.

The authors are currently studying ways to apply machine learning and other AI techniques to improve this research. Results showed that the new systems performed satisfactorily.

\section{Future Work}

Future work will consider the application of machine learning techniques on the collected data set to train an intelligent system to predict driving patterns, assess driving skills progress and analyze ability factors denoting the ability of a user to operate their wheelchair.

Future work will also consider adding a new function to the Python program to indicate if an intervention by the helper/carer or from a collision avoidance device occurred during a driving session, This will be used to provide a distinction of who is operating the device and providing information regarding driving ability and the need for assistance factors. Data collected will also be linked to driving and environmental situations and the targeted activity.

Finally, some decision making systems will be applied to the data [26-28]. Systems are clinically trialed at Chailey Heritage School as part of the bigger project [1].

\section{Acknowledgment}

Research in this paper was funded by EPSRC grant EP/S005927/1 and supported by The Chailey Heritage Foundation and the University of Portsmouth. 


\section{References}

1. Sanders, D. and Gegov, A.: Using artificial intelligence to share control of a powered-wheelchair between a wheelchair user and an intelligent sensor system. EPSRC Project 2019 - 2022, (2018).

2. Department for Work and Pensions 2017, accessed on 10 January 2020, <https://assets.publishing.service.gov.uk/government/uploads/system/upload s/attachment_data/file/692771/family-resources-survey-2016-17.pdf>

3. Disability facts and figures [Internet]. Accessed 2 December 2019. Available from: http://odi.dwp.gov.uk/disability-statistics-and-research/disability-factsand-figures.php.

4. Sanders, D. A.: Non-model-based control of a wheeled vehicle pulling two trailers to provide early powered mobility and driving experiences. IEEE Transactions on Neural Systems and Rehabilitation Engineering 26(1), 96 104 (2018).

5. Parhi, D. R. and Singh, M. K.: Rule-based hybrid neural network for navigation of a wheelchair, Proc. IMechE Part B: J. Engineering Manufacture 224, 11103 - 1117 (2009).

6. Sanders, D. A. Gegov, A. and Ndzi, D.: Knowledge-based expert system using a set of rules to assist a tele-operated mobile robot, in Studies in Computational Intelligence, (eds) Y. Bi, S. Kapoor, and R. Bhatia, 2018, 751, Springer, pp. 371-392 (2018).

7. Sanders, D. A. et al.: Rule-based system to assist a tele-operator with driving a mobile robot. Lecture Notes in Networks and Systems, 16, Springer, pp. 599-615 (2018).

8. Parhi D. R. et al.: The stable and precise motion control for multiple wheelchairs. Appl. Soft Comput 9(2), 477 - 487 (2009).

9. Nguyen, V. et al.: Strategies for Human - Machine Interface in an Intelligent Wheelchair, 35th Annual Int Conf of IEEE- (EMBC), Osaka, JAPAN. Book Series: IEEE Engineering in Medicine \& Biology Society Conf Proceedings, pp. 3638-3641 (2013).

10. Sanders, D, Langner, M, Bausch, N, Huang, Y, Khaustov, SA \& Simandjuntak, S.: Improving human-machine interaction for a powered wheelchair driver by using variable-switches and sensors that reduce wheelchair-veer. in Y BI, R Bhatia \& S Kapoor (eds), Intelligent Systems and Applications. Advances in Intelligent Systems and Computing, vol. 1038, Springer, pp. 1173-1191 (2019).

11. Okonor, OM, Gegov, A, Adda, M, Sanders, D, Haddad, MJM \& Tewkesbury, G.: Intelligent approach to minimizing power consumption in a cloud-based system collecting sensor data and monitoring the status of powered wheelchairs. in Y Bi, R Bhatia \& S Kapoor (eds), Intelligent Systems and Applications . Advances in Intelligent Systems and Computing, vol. 1037, Springer, pp. 694-710 (2019).

12. Sanders, D, Okonor, OM, Langner, M, Hassan Sayed, M, Khaustov, SA \& Omoarebun, PO.: Using a simple expert system to assist a powered wheelchair 
user. in $\mathrm{Y} \mathrm{Bi}$, R Bhatia \& $\mathrm{S}$ Kapoor (eds), Intelligent Systems and Applications. Advances in Intelligent Systems and Computing, vol. 1037, Springer, pp. 662-379 (2019).

13. Bausch, N, Shilling, P, Sanders, D, Haddad, MJM, Okonor, OM \& Tewkesbury, G.: Indoor location and collision feedback for a powered wheelchair system using machine learning. Advances in Intelligent Systems and Computing, Springer, IEEE SAI Intelligent Systems Conference, London, United Kingdom, vol. 1 pp. 721-739 (2019).

14. Tewkesbury, G, Sanders, D, Haddad, MJM, Bausch, N, Gegov, A \& Okonor, OM.: Task programming methodology for powered wheelchairs. Advances in Intelligent Systems and Computing, Springer, IEEE SAI Intelligent Systems Conference 2019, London, United Kingdom, vol. 1 pp. 711-720 (2019).

15. Sanders, D, Tewkesbury, G, Parchizadeh, H, Robertson, JJ, Omoarebun, PO \& Malik, M.: Learning to drive with and without intelligent computer systems and sensors to assist. in K Arai, S Kapoor \& R Bhatia (eds), Advances in Intelligent Systems and Computing, vol. 868, Springer, pp. 1171-1181 (2019).

16. Sanders, D, Gegov, A, Haddad, M, Ikwan, F, Wiltshire, D \& Tan, YC.: A rulebased expert system to decide on direction and speed of a powered wheelchair. in K Arai, S Kapoor \& R Bhatia (eds), Advances in Intelligent Systems and Computing, vol. 868, Springer, pp. 822-838 (2019).

17. Sanders, D, Gegov, A, Tewkesbury, G \& Khusainov, R.: Sharing driving between a vehicle driver and a sensor system using trust-factors to set control gains. in K Arai, S Kapoor \& R Bhatia (eds), Advances in Intelligent Systems and Computing, vol. 868, Springer, pp. 1182-1195 (2019).

18. Sanders, D. A. et al:: Results from investigating powered wheelchair users learning to drive with varying levels of sensor support, in Proc SAI Intelligent System, London, U.K. pp. 241-245 (2017).

19. Haddad, MJM, Sanders, D, Gegov, A, Hassan Sayed, M, Huang, Y \& AlMosawi, M.: Combining multiple criteria decision making with vector manipulation to decide on the direction for a powered wheelchair. in $\mathrm{Y} \mathrm{Bi}, \mathrm{R}$ Bhatia \& S Kapoor (eds), Advances in Intelligent Systems and Computing, vol. 1037, Springer, pp. 680-693 (2019).

20. Haddad, M, Sanders, D, Bausch, N, Tewkesbury, G, Gegov, A \& Hassan Sayed, M.: Learning to make intelligent decisions using an Expert System for the intelligent selection of either PROMETHEE II or the Analytical Hierarchy Process. in K Arai, S Kapoor \& R Bhatia (eds), Advances in Intelligent Systems and Computing, vol. 868, Springer, pp. 1303-1316 (2019).

21. Haddad, MJM \& Sanders, D.: Selecting a best compromise direction for a powered wheelchair using PROMETHEE. IEEE Transactions on Neural Systems and Rehabilitation Engineering 27(2), 228-235 (2019).

22. Haddad, MJM, Sanders, D, Tewkesbury, G, Gegov, A, Hassan Sayed, M \& Ikwan, FC.: Initial results from using Preference Ranking Organization METHods for Enrichment of Evaluations to help steer a powered wheelchair. in Y Bi, R Bhatia \& S Kapoor (eds), Advances in Intelligent Systems and Computing, vol. 1037, Springer, pp. 648-661 (2019). 
23. Sanders, D, Wang, Q, Bausch, N, Huang, Y, Khaustov, SA \& Popov, I, A method to produce minimal real time geometric representations of moving obstacles. in K Arai, S Kapoor \& R Bhatia (eds), Advances in Intelligent Systems and Computing, vol. 868, Springer, pp. 881-892 (2019).

24. Song, K. T. and Chen, C. C.: Application of asymmetric mapping for wheelchair navigation using ultrasonic sensors. J. Intell. Wheelchair Syst., 17(3), 243 - 264, (1996).

25. Sachdeva, P. and Katchii, S.: A Review Paper on Raspberry Pi. International Journal of Current Engineering and Technology 4(6), 3818 - 3819 (2014).

26. Haddad, MJM, Sanders, D \& Bausch, N.: Selecting a robust decision making method to evaluate employee performance. International Journal of Management and Decision Making, 18(4), 333-351 (2019).

27. Haddad, MJM, Sanders, D \& Tewkesbury, G.: Selecting a discrete Multiple Criteria Decision Making method to decide on a corporate relocation. Archives of Business Research 7(5), 48-67 (2019).

28. Sanders, D, Robinson, DC, Hassan Sayed, M, Haddad, MJM, Gegov, A \& Ahmed, N, Making decisions about saving energy in compressed air systems using Ambient Intelligence and Artificial Intelligence. in K Arai, S Kapoor \& R Bhatia (eds), Intelligent Systems and Applications: Proceedings of the 2018 Intelligent Systems Conference (IntelliSys) Volume 2. Advances in Intelligent Systems and Computing, vol. 869, Springer, pp. 1229-1236 (2019). 